\title{
Machine Learning for Stellar Magnetic Field Determination
}

\author{
J.P. Córdova Barbosa ${ }^{1}$, S.G. Navarro Jiménez ${ }^{2}$, J.C. Ramírez Vélez ${ }^{3}$ \\ ${ }^{1}$ CUCEA, Universidad de Guadalajara, \\ Periférico Norte 799, Núcleo Universitario Los Belenes, \\ Zapopan, Jalisco 45100, México \\ E-mail: jcordoba@astro.unam.mx \\ 2 Instituto de Astronomía y Metereología, Universidad de Guadalajara, \\ Av. Vallarta 2602, Arcos Vallarta Sur, \\ Guadalajara, Jalisco 44130, México \\ E-mail: silvana@astro.iam.udg.mx \\ ${ }^{3}$ Instituto de Astronomía Ensenada, Universidad Nacional Autónoma de México, \\ Carretera Tijuana-Ensenada km 103, Pedregal Playitas, \\ Ensenada, Baja California 22860, México \\ E-mail: jramirez@astro.unam.mx
}

Received 3 May 2017

Accepted 5 January 2018

\begin{abstract}
In this work we present the results for the automatic determination of the mean longitudinal magnetic field in polarized stellar spectra through the analysis of spectropolarimetric observations. In order to determine this important parameter, we first developed a synthetic database encompassing a set of different stellar spectra, each one defined by a set of free parameters. Then, we used supervised learning for artificial neural networks, a machine learning approach, to achieve our goal.
\end{abstract}

Keywords: Artificial Neural Networks, Machine Learning, Stellar: Magnetic Fields, Parameter Determination

\section{Introduction}

Nowadays there are plenty of astronomical databases available, ${ }^{1,2,3,4}$ containing enormous quantities of data, both real and synthetic. Hence, analysis and automatic extraction of relevant information from these collections have become an important task. Although there have been some successful efforts to retrieve some parameters from these databases, ${ }^{5,6,7}$ magnetic fields are a particularly complex phenomena and since they cannot be directly measured, their accurate determination is remarkably difficult. ${ }^{8}$ As a rule of thumb, magnetic fields are measured via the effects of their presence on other observable properties. The most successful method used to detect magnetic fields relies on the Zeeman effect. ${ }^{9}$ This effect is, essentially, the splitting of a spectral line due to the distortion of electron orbitals as a result of the presence of a magnetic field. This distortion depends on the quantum numbers of the energy level and the magnetic field intensity. ${ }^{10}$ On a spectrum formed in a region permeated by a magnetic field, the orbital energies between transitions are disturbed and, therefore, the 
absorption or emission lines might be affected.

The energy for each level is affected by the presence of a magnetic field, and each energy level with quantum number $\mathbf{J}$ splits into $(2 J+1)$ states of energy with different magnetic quantum numbers M. The difference between successive energy states $(\Delta E)$ is proportional to the magnetic field and to the Landé factor $(\mathbf{g})$, which is a function of the orbital angular momentum $(\mathbf{L})$ and the electron spin $(\mathbf{S})$ as described in Eq. (1).

$$
g_{i}=\frac{3}{2}+\frac{S_{i}\left(S_{i}+1\right)-L_{i}\left(L_{i}+1\right)}{2 J_{i}\left(J_{i}+1\right)} .
$$

Energy shifts are given by:

$$
\Delta E=\mu_{B} B M g
$$

Where $\mu_{B}$ is the Bohr magneton, $\mathrm{B}$ is the magnetic field strength, and $\mathrm{M}$ ranges from - $\mathrm{J}$ to $\mathrm{J}$, hence the previously mentioned $(2 J+1)$ different states. In the absence of a magnetic field, a transition between two levels, $E_{1}$ and $E_{2}$, with Landé factors $g_{1}$ and $g_{2}$ is characterized by a single energy level: $E_{2}-E_{1}$, but when a magnetic field is applied, the spectral line splits into closely dispersed segments with energies shifted from the original energy by:

$$
\begin{aligned}
\Delta E_{B} & =\mu_{B} B M_{2} g_{2}-\mu_{B} B M_{1} g_{1} \\
& =\mu_{B} B \cdot\left(M_{2} g_{2}-M_{1} g_{1}\right) .
\end{aligned}
$$

Considering $\Delta g=g_{2}-g_{1}$ and $\Delta M=M_{2}-M_{1}$ we can transform Eq. (3) as:

$$
\Delta E_{B}=\mu_{B} B \cdot\left(\Delta g M_{2}+\Delta M g_{1}\right) .
$$

A dipole transition between levels adheres to the selection rule $\Delta M=-1,0,1$ and therefore, the resulting spectral lines assemble into three groups; lines due to transitions with $\Delta M=0$, known as $\pi$ components and the groups of lines formed by transitions where $\Delta M= \pm 1$. The latter are known as $\sigma_{+}$ when lines are shifted to the right side in wavelength (red shifted) and $\sigma_{-}$, when they are blue shifted. Normally, both $\pi$ and $\sigma$ groups have several components, and when these components overlap, e.g. when $g_{2}=g_{1}$, the transition is called a Zeeman triplet. There are also some cases where lines show no splitting, e.g. when $g_{1}=0$ and $J_{2}=0$, known as magnetic null lines. Each group is characterized by different polarization states ${ }^{11}$ and their observed intensity depends on the angle between the line of sight and the magnetic field, among other parameters. Therefore, the measurement of the intensity at different polarized states is crucial for the proper characterization of a magnetic field.

The Stokes parameters ${ }^{12}$ is a widely used representation of polarized states. These parameters are a set of four values, named Stokes I,Q,U and $\mathrm{V}$, that describe the polarization state of electromagnetic radiation ${ }^{11}$ as follows; Stokes I is the integrated (non polarized) light, Stokes Q and U measure the two directions of linear polarization, and Stokes V measures circular polarization. These parameters are useful in astronomy because both circular and linear polarization states can be measured through appropriate instruments such as polarimeters and spectropolarimeters.

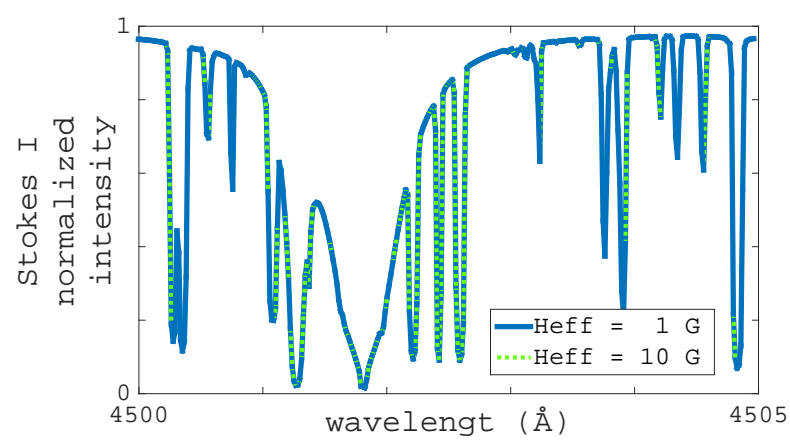

Fig. 1. Fragment of the spectra of the Stokes I parameter for a synthetic object simulated with different magnetic field.

Observations from Stokes I can be used to infer the magnetic field strength if for any given line, we can observe and measure the Zeeman splitting between the $\pi$ and $\sigma$ components of the transition. However, other broadening effects -as those induced by rotation, pressure, temperature, and otherscan mask the split of the Zeeman components. ${ }^{8}$ As a result, this technique is useful to measure only very strong magnetic fields, in the order of $10^{3}$ Gauss (kG) and higher, ${ }^{13}$ and becomes impractical for weaker fields. To illustrate this, the comparison between two Stokes I spectra for an object simulated with two different values of the effective magnetic field $\left(H_{e f f}\right), 1 \mathrm{G}$ and $10 \mathrm{G}$, is shown in Fig. 1. It 
is clear that the difference between both spectra is practically indistinguishable, emphasizing the complexity of the magnetic field measurements using only the Stokes I parameter.

Observations from the rest of the Stokes parameters (Q,U and V) are important because they are more sensitive to weaker fields due to the lower influence of other, non-magnetic effects. However, linear polarization produced by magnetic fields is generally weak, so its measurement in stars (associated with Stokes Q and U) is not common. ${ }^{8}$ Therefore, magnetic field measurements are typically performed using only Stokes I and Stokes V parameters. The Stokes V spectra of the same object depicted in Fig. 1 is shown in Fig. 2. It is clear that the difference between the two cases (1G and 10G) becomes more evident using this parameter.

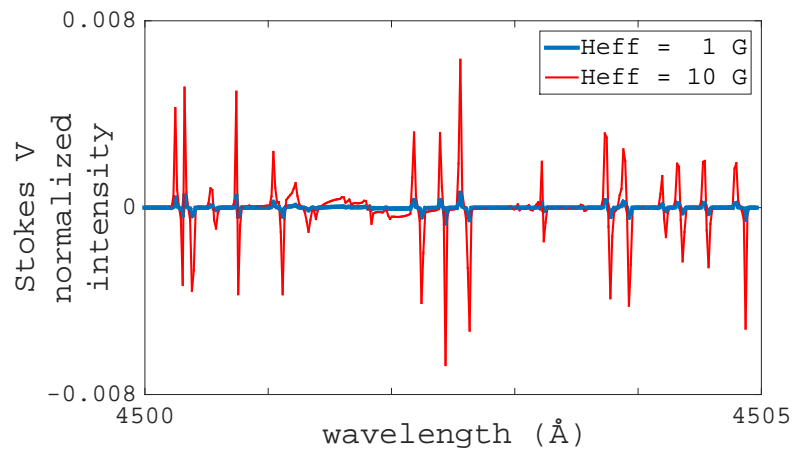

Fig. 2. Fragment of the spectra of the Stokes $\mathrm{V}$ parameter for a synthetic object simulated with different $H_{e f f}$.

Although the use of Stokes V allows to measure the magnetic field, it is known that, particularly on solar-type stars, magnetic fields are commonly found in the order of $10 \mathrm{G},{ }^{14}$ at which level the normalized intensity of this parameter is below the noise level. Looking to overcome this problem, several "multi-line" techniques have been developed. These techniques receive their name from the fact that they perform the addition of multiple individual lines on velocity domain, ${ }^{15}$ resulting in a mean profile or signature, known as multi-Zeeman Signatures (MZS). They encompass all the information from the polarized spectra, decreasing the noise level and at the same time performing a dimensionality data reduction. The most popular of these techniques is the Least Square Deconvolution (LSD) proposed by Donati et al. in Ref. 16, used to measure magnetic fields in several research papers. ${ }^{17,18,19,20}$

Nonetheless, looking to overcome the restrictions on the LSD method (assumptions of similarity between individual polarized circular lines and Weak Field Approximation) a different technique based on Principal Component Analysis (PCA) has been developed. This technique has been numerically validated to increase the Signal to Noise Ratio (SNR) of the MZS. ${ }^{21,22}$

\section{Methods}

In order to perform the automatic determination of the mean longitudinal magnetic field $\left(H_{e f f}\right)$ from polarized stellar spectra, the use of supervised $\mathrm{Ar}$ tificial Neural Networks (ANN) trained with a synthetic database of Multi-Zeeman Signatures (MZS) was proposed.

To create this database, it is necessary to model the Stokes parameters. This implies solving a set of four coupled first-order linear differential equations, ${ }^{23}$ each one corresponding to a single Stokes parameter. To address the problem that represents the computing needed to solve this system of equations, known as "Polarized Radiative Transfer (PRT) problem", several codes have been developed. ${ }^{24,25,26}$

In this work, the use of the COSSAM code was selected. COSSAM stands for "COdice per la Sintesi Spettrale nelle Atmosfere Magnetiche" or Code for the spectral synthesis in magnetic atmospheres. It was introduced in its latest form by Stift in Ref. 25. This object-oriented parallel code allows to accurately calculate the Stokes parameters for stars in which their magnetic field is represented by a tilted, eccentric dipole on assumed Local Thermodynamic Equilibrium (LTE). ${ }^{27}$

In order to calculate the Stokes parameters through COSSAM, it is necessary to define the following physical characteristics of the simulated object:

(i) Effective temperature $\left(T_{\text {eff }}\right)$

(ii) Surface gravity (expressed as $\log g$ ) 
(iii) Macro-turbulent velocity $\left(V_{t u r b}\right)$

(iv) Metallicity $[\mathrm{M} / \mathrm{H}]$

(v) Atomic transitions (taken from VALD: Vienna Atomic Line Database ${ }^{28}$ )

(vi) Micro turbulent velocity $(\xi)$

(vii) Inclination angle $(\boldsymbol{i})$, is the orientation of the rotational axis with respect to the Line Of Sight (LOS)

(viii) Orientation of the magnetic axis with respect to the rotation axis (described by the Euler Angles)

(ix) Position of the magnetic dipole inside the star (expressed by two coordinates: $x_{1}$ and $x_{2}$ )

(x) Dipole moment $(\boldsymbol{m})$

(xi) Projected rotational velocity $(v \sin i)$

(xii) Rotational phase

(xiii) Pulsational velocity and phase

(xiv) Spatial Grid

The first four characteristics $\left(T_{\text {eff }}, \log g, V_{\text {turb }}\right.$ and metallicity) define the commonly named "atmospheric model". Although, all the previously listed characteristics affect the behavior of the $H_{\text {eff }}$ to some degree, ${ }^{25}$ as a first step in our research, it was decided to limit the scope of this paper to only the case where all of the parameters are kept constant except for: $T_{\text {eff }}, \log g, V_{t u r b}$ and $\boldsymbol{m}$.

In order to build the database, first the widely employed Castelli \& Kurucz atmospheric models ${ }^{29}$ were obtained, then the ATLAS12ada ${ }^{30}$ code was used to transform them into the required format. Each of these models matches a different combination of the previously mentioned characteristics: $T_{e f f}, \log g$ and $V_{t u r b}$. For spectra synthesis, only solar metallicity was considered. Next, COSSAM was used employing a dipole centered magnetic model ${ }^{31}$ and keeping the spatial grid reduced to its simplest form: a single point at the center of the star.

To summarize, our database is integrated by varying the previously mentioned characteristics as shown in Table 1. It is significant to notice that the actual number of atmospheric templates (43) differs from the number expected according to Table 1 $(5 * 5 * 2=50)$, because some of the Kurucz templates were not available. It is also important to take into account that $H_{\text {eff }}$ varies at two different rates: first, from 1 to $20 \mathrm{G}$ at a $1 \mathrm{G}$ increment (20 steps), and then from 25 to $200 \mathrm{G}$ at a $5 \mathrm{G}$ increment (36 steps) resulting in 56 steps.

The total number of stellar spectra from the combination of all the parameters is $2048(43 * 56)$.

Table 1. Database characteristic information

\begin{tabular}{ccccc}
\hline Characteristic & First Value & Step & Final Value & Total \\
\hline \multicolumn{5}{c}{ Atmospheric Models } \\
$T_{\text {eff }}$ & 5000 & 500 & 7000 & 5 \\
$\log \mathrm{g}$ & 1 & 1 & 5 & 5 \\
$V_{\text {turb }}$ & 0 & - & 2 & 2 \\
\hline$H_{\text {eff }}$ & 1 & 1 & 20 & \\
& 25 & 5 & 200 & 56 \\
\hline
\end{tabular}

It is important to note that all the parameters that define the magnetic geometry were kept constant, and therefore, the data generated with COSSAM consists of Stokes I and V parameters only.

In order to make the spectra in our database closer to real spectra, which always contains noise, different levels of additive white Gaussian noise were incorporated to the Stokes V vectors $(5 \%, 10 \%$, $20 \%$ and $30 \%$ ) of the simulated (clean) spectra. A small fragment of the resulting noisy spectra for each case is shown in Fig. 3. These Noise Percentages (NP) were calculated as:

$$
N P=100 * \frac{\text { Noise Amplitude }}{\text { Spectrum Amplitude }} .
$$

Following this definition and considering that a standard SNR is defined as:

$$
S N R=\left(\frac{\text { Signal Amplitude }}{\text { Noise Amplitude }}\right)^{2}
$$

then we can go from NP to SNR, and viceversa, using:

$$
S N R=\left(\frac{100}{N P}\right)^{2}
$$




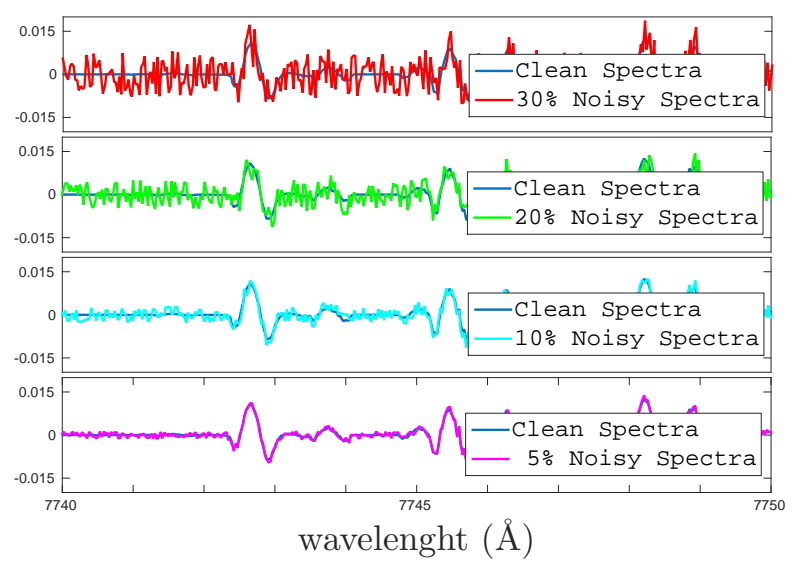

Fig. 3. Fragment of the noisy spectra of the Stokes V parameter.

According to Eq. (7), our NP levels turn into SNR as: 400, 100, 25 and $11 . \overline{11}$ for the Stokes V parameter. The set of 2048 clean spectra and its corresponding noisy spectra, all four NP/SNR levels, were accumulated in a bigger database consisting of 10,240 spectra. Next, PCA and Zeeman Doppler Imaging (ZDI) multi-line technique was used to obtain the $\mathrm{MZS}^{32}$ for each of the clean and noisy spectra. PCA is a standard technique used to extract relevant information from big, complex data sets and, at the same time, to reduce its dimensionality. It has been employed for several purposes, including; phase reconstruction, ${ }^{33}$ image noise level estimation, ${ }^{34}$ demodulation on interferometry, ${ }^{35}$ detection of exo-planets ${ }^{36}$ and more. ZDI is a tool frequently used to study stellar magnetic fields. ${ }^{20,37,38}$

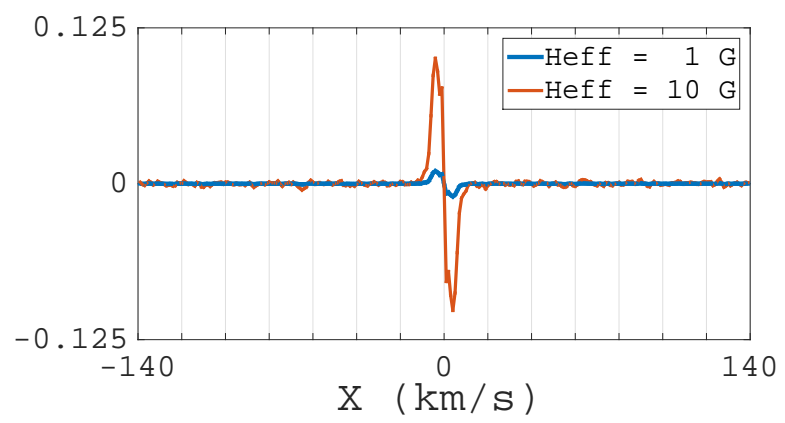

Fig. 4. MZS comparison for two synthetic objects with different $H_{\text {eff }}$.
To create the polarized signatures (MZS) from Stokes V, only the first component of PCA was used. The length of the MZS was set to 279 points, ranging from -139 to $139 \mathrm{~km} / \mathrm{s}$. Two examples of the resulting MZS are shown in Fig. 4. From this figure, it is evident that most of the information is contained in a smaller range (in this case, between -20 and 20 $\mathrm{km} / \mathrm{s}$ ).

The final database, used to train an ANN to determine $H_{\text {eff }}$, includes 10,240 of these Stokes V signatures, corresponding to both clean and noisy spectra. Stokes I signatures might be useful to determine other parameters $\left(T_{e f f}, \log g\right.$, etc. $)$.

After the evaluation of different architectures, the chosen ANN consists of a fully connected network with just one hidden layer. The input layer has 279 neurons (each one corresponding to a point from the MZS), the hidden layer has 10 neurons and the output layer has one neuron. In order to train the ANN, several training algorithms for the noise-free case were tested. For the noisy case, the algorithm with the best perfomance was chosen to train the final ANN. To validate the performance of each ANN, a $k$-fold cross validation process ${ }^{39}$ with $k=5$ was performed.

\section{Results and Discussion}

The mean measures of dispersion from the $\mathrm{k}$-fold cross validation of each ANN using only clean signatures are shown in Table 2; each one of the acronyms for the algorithms (listed on the header) stands for:

- bfg: BFGS quasi-Newton BackPropagation (BP)

- br : Bayesian regularization

- cgb: Powell-Beale Conjugate Gradient (CJ) BP

- cgf: Fletcher-Powell CJ BP

- cgp: Polak-Ribiere CJ BP

- gda: Gradient Descent (GD) with adaptive lr BP

- gdx: GD with momentum \& adaptive lr BP

- Im : Levenberg-Marquardt BP

- oss: One Step Secant

- rp : Resilient BP

- scg: Scaled Conjugate Gradient 
In the case of added noise to the stellar spectra it was decided, based on the measurements from table 2, to use Bayessian Regularization. ${ }^{40}$ This algorithm has both the best correlation coefficient (R) as well as the best Root Mean Square Error (RMSE).

From here on, all the results shown correspond to the final database (including both noisy and clean spectra). The regression performance for $H_{e f f} \mathrm{ob}-$ tained with the ANN is shown in Fig. 5. The Percentage Errors $^{41}(\mathrm{PE})$ from this regression have a normal distribution with $\mu=-1.5135 \%$ and $\sigma=$ $7.2836 \%$. The histogram that corresponds to these errors and their respective Probability Distribution Function (PDF); calculated using the normfit function from Matlab R2015a, is shown in Fig. 6. It is important to notice how the calculated PDF (shown as a continuous red line) seems to highly underestimate the real performance of the ANN. This effect is produced by the high percentage errors found for the lowest fields, around 1G. If the PDF is calculated dismissing these errors, i.e. higher than $20 \%$, then a different PDF more faithful to the actual distribution of the histogram can be found. This PDF, with $\mu=-0.5937 \%$ and $\sigma=3.9310 \%$, is also shown in Fig. 6 as a dashed yellow line. Further statistical measures of the regression performance are shown in Table 3.

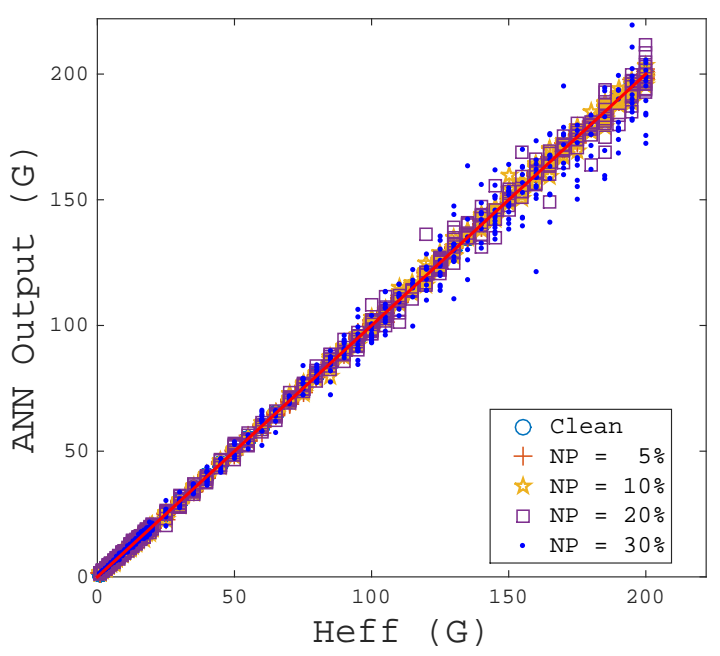

Fig. 5. $H_{\text {eff }}$ Regression plot.
As expected, the cases with the highest absolute error $^{41}$ are those where the noise is higher. Most of them occur when $N P=30 \%$, which also means the lowest SNR, as shown in Fig. 5.

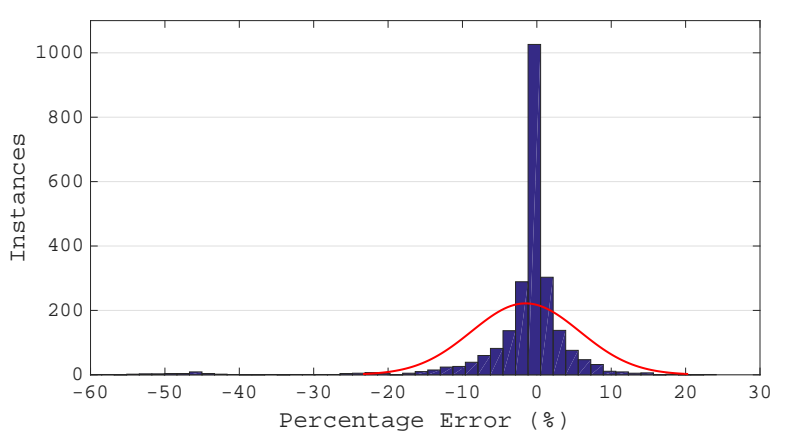

Fig. 6. Percentage Errors Histogram and PDF.

The behavior of both absolute and percentage errors related to $H_{\text {eff }}$ is shown in Figures Fig. 7(a) and Fig. 7(b) respectively. From the upper panel, it is clear that as the field intensity increases so does the absolute error. However, from the bottom panel, corresponding to percentage errors, it is noteworthy that they seem to be constant along the full range, except for the cases where $H_{e f f}$ is closer to zero. Nonetheless, this is expected because of the formula used to calculate them.

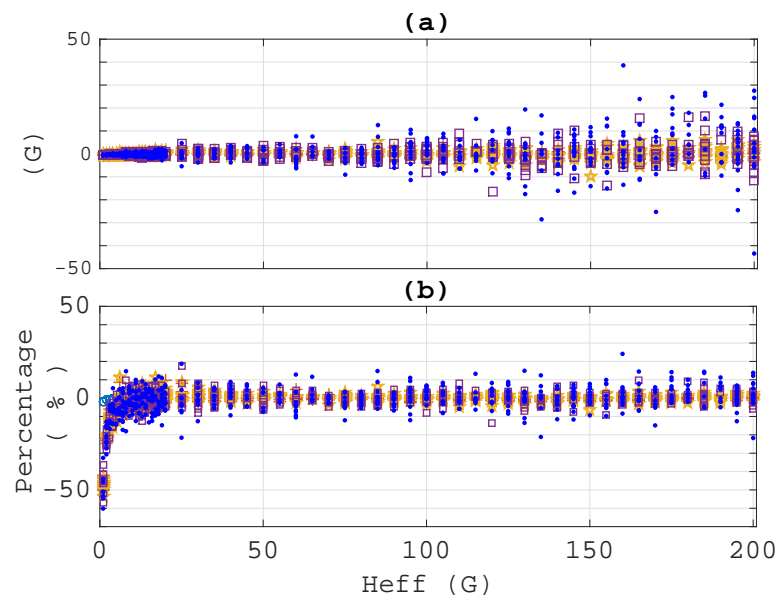

Fig. 7. $H_{\text {eff }}$ (a) Absolute Error and (b) Percentage Error.

Notice that the same symbols used in Fig. 5 to denote NP levels are used in both Figures 7(a) and 
Table 2: Training Algorithms: Mean Measures of Dispersion

\begin{tabular}{cccccccccccc}
\hline & bfg & br & cgb & cgf & cgp & gda & gdx & lm & oss & rp & scg \\
\hline R & 0.9876 & $\mathbf{0 . 9 9 8 3}$ & 0.9982 & 0.9975 & 0.9975 & 0.9818 & 0.9787 & 0.9982 & 0.9972 & 0.9954 & 0.9974 \\
MSE & 101.923 & 13.618 & 15.148 & 20.547 & 20.265 & 150.93 & 175.822 & $\mathbf{1 3 . 3 8 3}$ & 23.193 & 37.463 & 21.621 \\
RMSE & 9.9902 & $\mathbf{3 . 6 6 5 1}$ & 3.8898 & 4.5279 & 4.4930 & 12.2596 & 13.1695 & 3.6743 & 4.8031 & 6.0966 & 4.6339 \\
RRSE & 0.1558 & $\mathbf{0 . 0 5 7 1}$ & 0.0606 & 0.0706 & 0.0700 & 0.1912 & 0.2053 & 0.0578 & 0.0749 & 0.0952 & 0.0723 \\
MAE & 5.7469 & 1.8140 & 2.3632 & 2.7658 & 2.6847 & 7.5064 & 7.6297 & $\mathbf{1 . 7 7 9 6}$ & 2.9001 & 3.6230 & 2.7722 \\
RAE & 0.1011 & 0.0319 & 0.0415 & 0.0486 & 0.0471 & 0.1319 & 0.1341 & $\mathbf{0 . 0 3 1 2}$ & 0.0509 & 0.0638 & 0.0487 \\
\hline
\end{tabular}

Table 3: $H_{e f f}$ regression: Statistical results

\begin{tabular}{ccccccc}
\hline & Clean & Noisy 5\% & Noisy 10\% & Noisy 20\% & Noisy 30\% & Combined \\
\hline R & 1 & 0.9997 & 0.9981 & 0.9957 & 0.9919 & 0.9983 \\
MSE & $0.37 \times 10^{-3}$ & 2.2525 & 15.4002 & 35.7429 & 66.6803 & 13.6181 \\
\hline RMSE & 0.0180 & 1.4991 & 3.9234 & 5.9735 & 8.1564 & 3.6651 \\
RRSE & $0.28 \times 10^{-3}$ & 0.0234 & 0.0612 & 0.0932 & 0.1273 & 0.0572 \\
MAE & 0.0151 & 0.9294 & 2.2574 & 3.4641 & 4.6954 & 1.8141 \\
RAE & $0.26 \times 10^{-3}$ & 0.0164 & 0.0397 & 0.0609 & 0.0826 & 0.0319 \\
\hline
\end{tabular}

7(b). As expected, the spectra with the highest noise produce the cases with the highest errors. Following the calculated Normal distribution probability for the percentage errors, as shown in Fig. 6, the probability of having a percentage error between $10 \%$ and $-10 \%$ is $P(-10 \%<P E<10 \%)=82.11 \%$, which improves to $P(-15 \%<P E<15 \%)=95.63 \%$ and $P(-20 \%<P E<20 \%)=99.29 \%$.

Based on the results shown above, it can be concluded that the use of Machine Learning, specifically Artificial Neural Networks, allows for the determination of the mean longitudinal magnetic field of stars. This can be achieved within an $\pm 20 \%$ margin of error $99 \%$ of the times for $H_{\text {eff }}>1 G$, even when the Signal to Noise Ratio of the spectra is as low as 11 .

The results obtained in this work already represent an important development in the application of ANN on real data. However, our next goal is to expand the database to include variations in all of the parameters that remained fixed in this work, in order to obtain the $H_{\text {eff }}$ from real objects. Nevertheless, in this paper, the use of machine learning algorithms has been proven to be a powerful tool in the study of magnetic fields through the analysis of polarized spectra.

\section{Acknowledgments}

Part of the results presented here were obtained using the "UNAM Supercómputo - DGTIC" facilities, grant SC16-1-IR-40, the computers from projects CONACyT 153985 and UNAM-PAPIIT 107215 and the computers "Tycho" (Posgrado en Astrofísica, Instituto de Astronomía-UNAM and PNPC-CONACyT).

J.P. Córdova acknowledges the Mexican National Council for Science and Technology (CONACyT) for its financial support, J.C. Ramírez and S.G. Navarro acknowledge financial support from CONACyT grants 240441 and 168078 respectively. 


\section{References}

1. P. Petit et al., PolarBase: A Database of HighResolution Spectropolarimetric Stellar Observations, PASP 126 (2014), pp. 469-475.

2. A. Dotter et al., The Dartmouth Stellar Evolution Database, ApJS 178 (2008), pp. 89-101.

3. X. Haubois et al., A global database for optical interferometry, in Proceedings SPIE 9146 (2014).

4. L. Beitia-Antero and A.I. Gómez de Castro, A database of synthetic photometry in the GALEX ultraviolet bands for the stellar sources observed with the International Ultraviolet Explorer, A\&A 596 (2016).

5. H. Posbic et al., SPADES: a stellar parameters determination software, $A \& A \mathbf{5 4 4}$ (2012).

6. A. Mucciarelli et al., GALA: An automatic tool for the abundance analysis of stellar spectra, ApJ 766 (2013).

7. L. Sbordone et al., MyGIsFOS: an automated code for parameter determination and detailed abundance analysis in cool stars, $A \& A 564$ (2014).

8. A. Reiners, Observations of Cool-Star Magnetic Fields, in Living Reviews in Solar Physics 9 (2012).

9. P. Zeeman, On the Influence of Magnetism on the Nature of the Light Emitted by a Substance, ApJ 5 (1897).

10. J.F. Donati and J. Landstreet, Magnetic Fields of Nondegenerate Stars, ARA\&A 47 (2009), pp. 333-370.

11. E. Collett, Field guide to polarization, (SPIE Press, 2005).

12. G.G. Stokes, On the Change of Refrangibility of Light, in Philosophical Transactions of the Royal Society of London 142 (1852), pp. 463-562.

13. J. Robinson et al., Magnetic field measurements on stellar sources - A new method, Apj 239 (1980).

14. S.C. Marsden et al., A BCool magnetic snapshot survey of solar-type stars, MNRAS 444 (2014), pp. 35173536.

15. M. Semel and J. Li, Zeeman-Doppler Imaging of Solar-Type Stars: Multi Line Technique, SoPh 164 (1996), pp. 417-428.

16. J.F. Donati et al., Spectropolarimetric observations of active stars, MNRAS 291 (1997), pp. 658-682.

17. E. Alecian et al., A high-resolution spectropolarimetric survey of Herbig Ae/Be stars - I. Observations and measurements, MNRAS 429 (2013), pp. 1001-1026.

18. A. Asensio Ramos and P. Petit, Bayesian least squares deconvolution, $A \& A \mathbf{5 8 3}$ (2015).

19. O. Kochukhov et al., Least-squares deconvolution of the stellar intensity and polarization spectra, $A \& A \mathbf{5 2 4}$ (2010).

20. L. Rosén et al., Magnetic fields of young solar twins, A\&A 593 (2016).

21. M. Semel et al., Multi-Line Spectro-Polarimetry of Stellar Magnetic Fields Using Principal Components Analysis, ASP Conf. Ser. 358 (2006), pp. 355-361.
22. J.C. Ramírez Vélez et al., Stellar longitudinal magnetic field determination through multi-Zeeman signatures, $A \& A \mathbf{5 9 6}$ (2016).

23. G.A. Wade et al., LTE spectrum synthesis in magnetic stellar atmospheres, $A \& A 374$ (2001), pp. 265-279.

24. N. Piskunov, INVERS10: A New Code for Magnetic Doppler Imaging, ASP Conf. Ser. 154 (1998).

25. M. Stift, COSSAM: Codice per la Sintesi Spettrale nelle Atmosfere Magnetiche, A Peculiar Newsletter 33 (2000).

26. S. Reissl et al., Radiative transfer with POLARIS, A\&A 593 (2016).

27. H.W. Drawin, Validity conditions for local thermodynamic equilibrium, Physik 228 (1969), pp. 99-119.

28. U. Heiter et al., VALD an atomic and molecular database for astrophysics, J Phys Conf Ser 130 (2008).

29. F. Castelli and R.L. Kurucz, New Grids of ATLAS9 Model Atmospheres, eprint arXiv:astro-ph/0405087 (2014).

30. K.M. Bischof, Two platform independent versions of ATLAS12, Memorie della Societá Astronomica Italiana Supplement 8 (2005).

31. M.J. Stift, A Non-Axisymmetric Rigid Rotator Model for Magnetic Stars, MNRAS 172 (1975), pp. 133-139.

32. J.C. Ramírez Vélez et al., Spectropolarimetric multiline analysis of stellar magnetic fields, $A \& A \mathbf{5 1 2}$ (2010).

33. J. Deng et al., Advanced principal component analysis method for phase reconstruction, Optics Express 23 (2015).

34. S. Pyatykh et al., Image Noise Level Estimation by Principal Component Analysis, IEEE Trans Image Process 22 (2013), pp. 687-699.

35. J. Vargas et al., Generalization of the Principal Component Analysis algorithm for interferometry, Optics Communications 286 (2013), pp. 130-134.

36. R. Soummer et al., Detection and characterization of exoplanets and disks using projections on KarhunenLoeve eigenimages, ApJ 755 (2012).

37. L. Rosén et al., First Zeeman Doppler Imaging of a cool star using all four stokes parameters, ApJ $\mathbf{8 0 5}$ (2015).

38. V. See et al., Studying stellar spin-down with ZeemanDoppler magnetograms, MNRAS 466 (2017), pp. 1542-1554.

39. J. Rodriguez et al., Sensitivity Analysis of k-Fold Cross Validation in Prediction Error Estimation, IEEE Trans. Pattern Anal. Mach. Intell. 32 (2010), pp. 569575.

40. B.P. Carlin and T.A. Louis, Bayesian methods for data analysis, $3^{\text {rd }}$ edn. (Chapman \& Hall/CRC, 2009).

41. M. Abramowitz and I.A. Stegun (eds.), Handbook of Mathematical Functions with Formulas, Graphs, and Mathematical Tables, $9^{\text {th }}$ printing. (Dover, New York, 1972). 